\title{
Pushing the Frontiers: uncovering the earliest galaxies in the Hubble Frontier Fields
}

\author{
Rachael C. Livermore \\ University of Texas at Austin, 2515 Speedway Stop C1400, Austin, TX 78741, USA \\ email: r.c.livermore@astro.as.utexas.edu
}

\begin{abstract}
With the combination of deep Hubble Space Telescope imaging and magnification due to gravitational lensing, the Hubble Frontier Fields program offers an unprecedented opportunity to study the faint end of the luminosity function at the highest redshifts. Unfortunately, the region of the field that benefits most from this magnification (the immediate vicinity of the critical line) suffers from a high abundance of bright foreground galaxies and high background due to intracluster light. To overcome these difficulties, some method of modelling and subtracting the foreground light is required. Here, I present results using wavelet decomposition to subtract large-scale structures in the clusters, which significantly increases the effective depth of the images, and crucially opens up the most magnified regions of the clusters.
\end{abstract}

Keywords. galaxies: high-redshift, galaxies: luminosity function, gravitational lensing

\section{Introduction}

Deep surveys with $H S T$ /WFC3 have enabled the detection of large numbers of highredshift galaxies, leading to great progress in our understanding of galaxies in the early Universe. Several different groups have independently characterised the rest-frame UV luminosity function during the epoch of reionization $(z \sim 7)$, finding a steep faint-end slope $(\alpha \sim-2$; Bouwens et al. 2011, Bouwens et al. 2015, McLure et al. 2013, Finkelstein et al. 2015). This implies that the UV luminosity density in this epoch is dominated by faint galaxies, and therefore that faint galaxies could be responsible for reionization. However, the faint end is highly uncertain due to the limited depth, and the faintest galaxies $\left(M_{1500} \sim-18\right)$ are all found in a single small field, the Hubble Ultra Deep Field, so cosmic variance is an issue. Furthermore, the energy required for reionization requires that we integrate the UV luminosity function below the observational limits. This requires the assumption that the faint-end slope remains steep beyond this limit, whereas simulations suggest there should be a turnover at some limiting halo mass (Finlator, Davé \& Özel 2011, Boylan-Kolchin, Bullock \& Garrison-Kimmel 2014, O'Shea et al. 2015)

The Hubble Frontier Fields (HFF) program (PI: Lotz) aims to use gravitational lensing to overcome this problem by observing six strongly lensing clusters to benefit from the magnification of background sources. Early work on these data has extended the UV luminosity function to $M_{1500} \sim-15$ (Atek et al. 2014,2015, Ishigaki et al. 2015, Laporte et al. 2015), but have been limited by the difficulty of detecting faint sources in the central regions of the cluster where there are a large number of foreground cluster galaxies and high background due to intracluster light. We therefore focus on removing this contamination in order to increase the yield of magnified high- $z$ galaxies. 


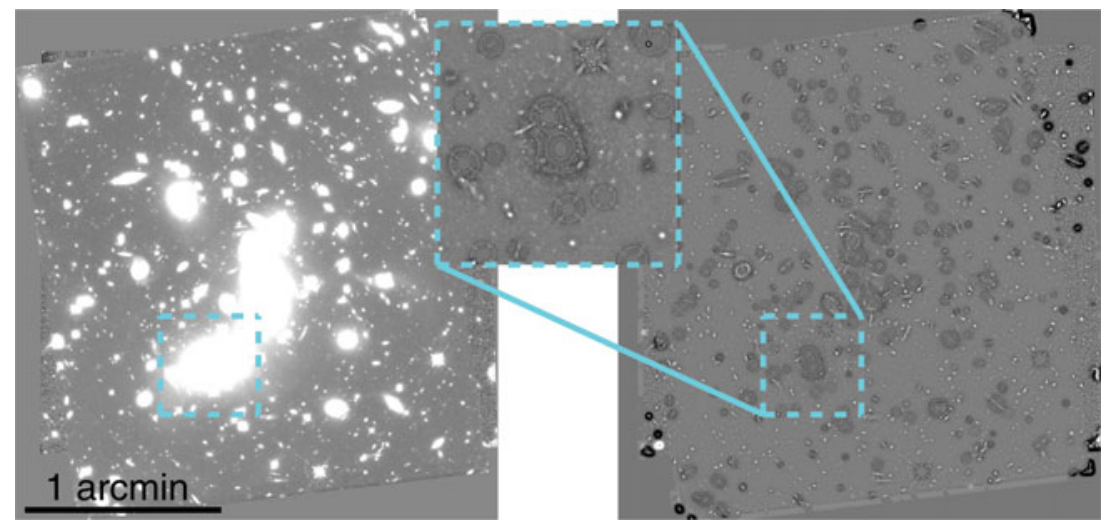

Figure 1. The $H_{160}$-band image of Abell 2744 before (left) and after (right) wavelet subtraction, shown with the same scaling. The cutout region of the wavelet-subtracted image highlights the small, faint sources that be found after subtraction of the large structures.

\section{Wavelet decomposition}

In order to overcome the difficulties inherent in detecting galaxies in the cluster center, we have developed a technique of removing the foreground galaxies and intracluster light using wavelet decomposition. The image is convolved with a wavelet function on a small scale; this convolved image is then subtracted from the original and the residuals are convolved with the same wavelet function on a larger scale. This process is repeated for ten physical scales, resulting in 10 convolved images, the sum of which is equal to the original image (Starck et al. 2002). We then detect objects in each of the 10 convolved images and trace them through the images to find the largest physical scale on which each is identified. For a given scale $n$, we subtract any pixels associated with objects detected on scales $\geqslant n$ from each image and then add them together to produce the wavelet-subtracted image. The result is shown in Figure 1.

We carry out extensive tests of the wavelet subtraction method, demonstrating that it enables detection of fainter galaxies with accurate photometry. In particular, this method almost eliminates the position-dependent variance in depth across the image, allowing us to achieve the full depth directly behind the cluster core. It should be noted that larger, brighter high- $z$ galaxies can be subtracted in the process; hence, we use both the original and wavelet-subtracted images for source selection.

\section{Source selection}

We PSF-match all images to the $H_{160}$-band and detect galaxies in the two cluster fields using Source Extractor (SExTRACTOR; Bertin \& Arnouts 1996) in dual image mode. We use 22 detection images in total, comprising all combinations of the WFC3 images in both the original and wavelet-subtracted forms. We measure photometry in the original images using a small (0.5") mesh for background subtraction. The 22 resulting catalogs are combined into a single master catalog using a matching radius of 0.2 ".

We perform photometric redshift fitting with EAZY (Brammer, van Dokkum \& Coppi 2008). This produces a redshift probability distribution function (PDF) for each galaxy, which we use to select the high- $z$ sample as follows: the integral under the primary peak of the PDF must be $>0.7$, so that we only select galaxies where one redshift is strongly preferred; the best-fit redshift must be $>4$, to ensure that the preferred redshift solution is the high- $z$ one; and the integral of the PDF in each redshift bin $(\delta z=1)$ must be 
Table 1. High- $z$ galaxy counts in the first two cluster fields

\begin{tabular}{l|cccc}
\hline Cluster & $z \sim 6$ & $z \sim 7$ & $z \sim 8$ & $z \sim 9$ \\
\hline Abell 2744 & 58 & 26 & 7 & 1 \\
MACS 0416 & 51 & 19 & 5 & 3 \\
\hline
\end{tabular}

$\geqslant 0.25$ in a single bin at $z \geqslant 6$ to exclude galaxies with very broad PDFs. The galaxy is then allocated to the redshift bin with the largest PDF integral.

We then compare the colors and radii of the bright $\left(H_{160} \leqslant 27\right)$ galaxies in the sample to those of M, L and T dwarfs, as these can be selected as high- $z$ galaxies. We find one source that matches the colors of dwarf stars and is unresolved, so we exclude this from our sample. The entirety of the remaining sample is then visually inspected to remove any image artefacts or spurious sources.

The final sample comprises 170 galaxies: 92 in Abell 2744 and 78 in MACS 0416. The counts in each redshift bin are listed in Table 1.

\section{Magnification}

We use the lens models of Jauzac et al. $(2015 \mathrm{a}, \mathrm{b})$ to calculate the magnification of each galaxy. We use the luminosity-weighted mean magnification across the image (from the SEXTRACTOR segmentation map), as this gives more physical magnification factors for galaxies that are very close to the critical line.

To estimate the error on the magnification, we use the errors in the convergence and shear maps (based on the range of alternate models provided by the lens modellers) and the $68 \%$ confidence range of the photo- $z$ from EAZY. The photo- $z$ error dominates the error in the magnification for all but the most highly-magnified sources.

\section{Completeness}

In order to compute the luminosity function, it is necessary to have a thorough understanding of the detection efficiency across the image. We carry out extensive simulations to characterize the completeness of our detection method.

In each iteration of the simulation, we generate 250 fake galaxy images. These galaxies are generated to have a range of randomly generated sizes, magnitudes and redshifts. We also generate random ages, dust attenuation factors $(\mathrm{E}(\mathrm{B}-\mathrm{V}))$ and metallicities, which are combined to form a distribution of UV slopes $\beta$. These properties are all combined using the stellar population models of Bruzal \& Charlot (2003) to generate galaxy colors. These are then scaled to the chosen $H_{160}$-band magnitudes to obtain magnitudes in the other six filters. These properties are all used to generate postage stamp images of fake galaxies using GALFIT (Peng et al. 2002).

We select random positions in the image plane and delens them to select source-plane positions to add these fake galaxy images; this ensures that the highly magnified region is overpopulated. We then lens the fake images, convolve the result with the $H_{160}$-band $\mathrm{PSF}$ and add the resulting image to the real cluster image in each filter. We then carry out the wavelet decomposition procedure and detect sources using the same procedure as that described above for the real data, including the fitting of photometric redshifts and photo- $z$ quality cuts. The resulting catalog is then compared to the input sources to determine the detection efficiency.

This process is repeated 2,000 times, resulting in 500,000 fake galaxies. This gives us sufficient statistics to determine the completeness fraction as a function of both 

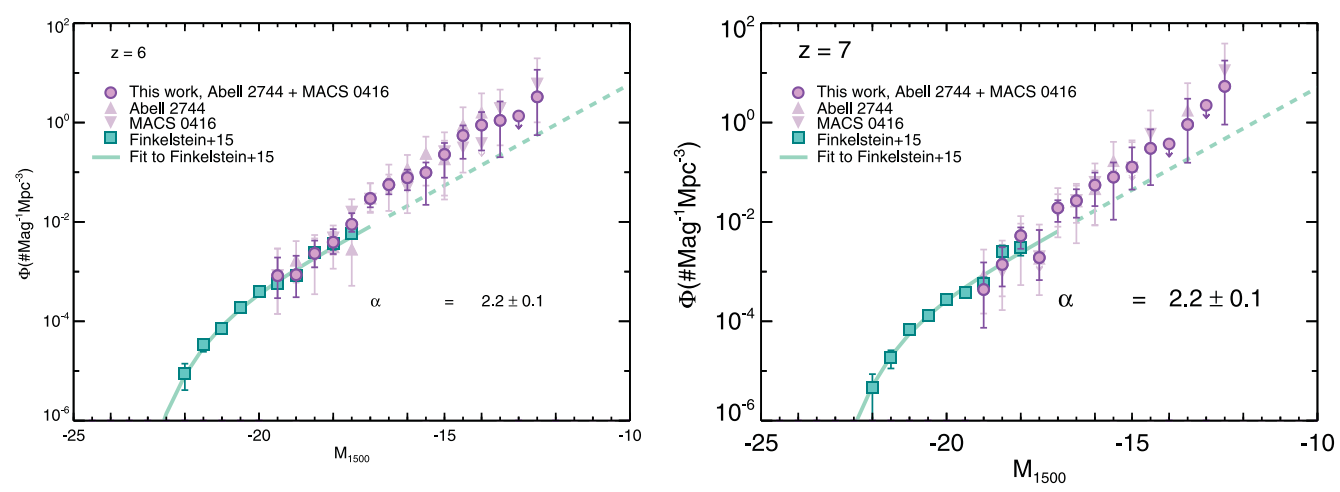

Figure 2. The luminosity functions at $z \sim 6$ (left) and $z \sim 7$ (right) at rest-frame $1500 \AA$. We find a steep faint-end slope possibly extending as far as $M_{1500}=-13$, although the volume in this regime is small.

position and magnitude. We then use this to calculate the effective volume as a function of magnitude, which can be used to calculate the intrinsic galaxy density.

\section{The UV Luminosity Function at $6<z<7$}

Using the effective volume as a function of magnitude calculate as described above, we produce the luminosity function at $z \sim 6-7$, shown in Figure 2. The error bars on $\phi$ are generated with a Monte Carlo procedure where galaxies' magnitudes are permitted to vary within the errors of their photometry and magnifications, resulting in their scattering between magnitude bins.

We find a steep faint-end slope $\alpha \sim-2.2$ which extends as faint as $M_{1500}=-13$. However, we note that there is a single galaxy in the faintest bin, and the volume in the faint regime is very small, being dependent only on the most magnified regions of the clusters. The full Hubble Frontier Fields program will offer significant improvement in the constraints at the faint end.

\section{References}

Atek, H. et al. 2014 ApJ, 786, 60

Atek, H. et al. 2015 ApJ 800, 18

Bertin, E. \& Arnouts, S. 1996, A\&AS, 117, 393

Bouwens, R. J. et al. 2011, ApJ, 737, 90

Bouwens, R. J. et al. 2015, ApJ, 803, 34

Boylan-Kolchin, M., Bullock, J. S., \& Garrison-Kimmel, S. 2014, MNRAS, 443, L44

Brammer, G. B., van Dokkum, P. G., \& Coppi, P. 2008, ApJ, 686, 1503

Bruzal, G. \& Charlot, S. 2003, MNRAS, 344, 1000

Finkelstein, S. L. et al. 2015, ApJ, 810, 71

Finlator, K., Davé, R., \& Özel, F. 2011, ApJ, 743, 169

Ishigaki, M. et al. 2015, ApJ, 799, 12

Jauzac, M. et al. 2015a, MNRAS, 446, 4132

Jauzac, M. et al. 2015b, MNRAS, 452, 1437

Laporte et al. 2015, A\&\&A, 575, 92

McLure, R. J. et al. 2013, MNRAS, 432, 2696

O'Shea, B. W., Wise, J. H., Xu, H., \& Norman, M. L. 2015, ApJL, 807, 12

Peng, C., Ho, L., Impey, C., \& Rix, H.-W. 2002, AJ, 124, 266

Starck, J.-L. et al. 2002, PASP, 114, 1051 